Bull. Austral. Math. Soc.

$53 \mathrm{~B} 20,53 \mathrm{c} 50,53 \mathrm{c} 42,53 \mathrm{c} 17$

VOL. 66 (2002) [177-191]

\title{
CONVOLUTION OF RIEMANNIAN MANIFOLDS AND ITS APPLICATIONS
}

\author{
BANG-YEN CHEN
}

\begin{abstract}
It is well-known that warped products play some important roles in differential geometry as well as in physics. In this article we extend the notion of warped product to the notion of convolution of Riemannian manifolds. We study the basic properties of convolutions of Riemannian manifolds. We also apply the notion of convolution to establish and characterise the Euclidean version of Segre embedding.
\end{abstract}

\section{Convolution of Riemannian manifolds}

Let $\left(N_{1}, g_{1}\right)$ and $\left(N_{2}, g_{2}\right)$ be two Riemannian manifolds and $f$ be a positive differentiable function on $N_{1}$. The well-known notion of warped product $N_{1} \times_{f} N_{2}$ is the product manifold $N_{1} \times N_{2}$ equipped with the warped product metric $g_{1}+f^{2} g_{2}$. It is well-known that the notion of warped products plays some important roles in differential geometry as well as in physics (see [7]).

The following notion of convolution of Riemannian manifolds extends the notion of warped products in a natural way. Let $\left(N_{1}, g_{1}\right)$ and $\left(N_{2}, g_{2}\right)$ be two Riemannian manifolds and let $f$ and $h$ be two positive differentiable functions on $N_{1}$ and $N_{2}$, respectively. Consider the symmetric tensor field ${ }_{h} g_{1} *_{f} g_{2}$ of type $(0,2)$ on $N_{1} \times N_{2}$ defined by

$$
{ }_{h} g_{1} *{ }_{f} g_{2}=h^{2} g_{1}+f^{2} g_{2}+2 f h d f \otimes d h
$$

The symmetric tensor field ${ }_{h} g_{1} *_{f} g_{2}$ is called the convolution of $g_{1}$ and $g_{2}$ via $h$ and $f$. The product manifold $N_{1} \times N_{2}$ together with ${ }_{h} g_{1} *_{f} g_{2}$, denoted by ${ }_{h} N_{1} \star_{f} N_{2}$, is called a convolution manifold. When $f, h$ are irrelevant, ${ }_{h} N_{1} \star{ }_{f} N_{2}$ and ${ }_{h} g_{1} * f g_{2}$ are simply denoted by $N_{1} \star N_{2}$ and $g_{1} * g_{2}$, respectively.

When ${ }_{h} g_{1} * f g_{2}$ is a positive-definite symmetric tensor, it defines a Riemannian metric on $N_{1} \times N_{2}$. In this case, ${ }_{h} g_{1} *_{f} g_{2}$ is called a convolution metric and the convolution manifold ${ }_{h} N_{1} \star{ }_{f} N_{2}$ is called a convolution Riemannian manifold.

In the first part of this article we show that the notion of convolution of Riemannian manifolds arises naturally. In the second part, we apply the notion of convolution to provide a fundamental study of the differential geometry of the tensor product $\mathbf{C}^{h} \otimes \mathbf{E}^{p}$. In particular, we apply the notion of convolution to establish and characterise the Euclidean version of Segre embedding.

Received 23rd July, 2001

Copyright Clearance Centre, Inc. Serial-fee code: 0004-9727/02 \$A2.00+0.00. 


\section{Preliminaries}

Let $N$ be a Riemannian manifold equipped with a Riemannian metric $g$. The gradient $\nabla \varphi$ of a function $\varphi$ on $N$ is defined by $\langle\nabla \varphi, X\rangle=X \varphi$ for vector fields $X$ tangent to $N$. If $N$ is a submanifold of a Riemannian manifold $\widetilde{M}$, the formulas of Gauss and Weingarten are given respectively by

$$
\begin{aligned}
\widetilde{\nabla}_{X} Y & =\nabla_{X} Y+\sigma(X, Y), \\
\widetilde{\nabla}_{X} \xi & =-A_{\xi} X+D_{X} \xi
\end{aligned}
$$

for vector fields $X, Y$ for vector fields $X, Y$ tangent to $N$ and $\xi$ normal to $N$, where $\tilde{\nabla}$ denotes the Riemannian connection on $\widetilde{M}, \sigma$ the second fundamental form, $D$ the normal connection, and $A$ the shape operator of $N$ in $\widetilde{M}$. The second fundamental form and the shape operator are related by $\left\langle A_{\xi} X, Y\right\rangle=\langle\sigma(X, Y), \xi\rangle$, where $\langle$,$\rangle denotes the inner$ product on $M$ as well as on $\widetilde{M}$. A submanifold in a Riemannian manifold is called totally geodesic if its second fundamental form vanishes identically.

The equation of Gauss of $N$ in $\widetilde{M}$ is given by

$$
\widetilde{R}(X, Y ; Z, W)=R(X, Y ; Z, W)+\langle\sigma(X, Z), \sigma(Y, W)\rangle-\langle\sigma(X, W), \sigma(Y, Z)\rangle,
$$

for $X, Y, Z, W$ tangent to $M$, where $R$ and $\widetilde{R}$ denote the curvature tensors of $N$ and $\widetilde{M}$, respectively.

The covariant derivative $\bar{\nabla} \sigma$ of $\sigma$ with respect to the connection on $T M \oplus T^{\perp} M$ is defined by

$$
\left(\bar{\nabla}_{X} \sigma\right)(Y, Z)=D_{X}(\sigma(Y, Z))-\sigma\left(\nabla_{X} Y, Z\right)-\sigma\left(Y, \nabla_{X} Z\right)
$$

The equation of Codazzi is

$$
(\widetilde{R}(X, Y) Z)^{\perp}=\left(\bar{\nabla}_{X} \sigma\right)(Y, Z)-\left(\bar{\nabla}_{Y} \sigma\right)(X, Z),
$$

where $(\widetilde{R}(X, Y) Z)^{\perp}$ denotes the normal component of $\widetilde{R}(X, Y) Z$.

Let $\mathbf{C}^{m} \otimes \mathbf{E}^{n}$ denote the tensor product of $\mathbf{C}^{m}$ and $\mathbf{E}^{n}$. Then $\mathbf{C}^{m} \otimes \mathbf{E}^{n}$ is holomorphically isometric to $\mathbf{C}^{m n}$. The inner product on $\mathbf{C}^{m} \otimes \mathbf{E}^{n}$ is given by

$$
\langle\alpha \otimes \beta, \gamma \otimes \delta\rangle=\langle\alpha, \gamma\rangle\langle\beta, \delta\rangle
$$

where $\langle\alpha, \gamma\rangle$ is the inner product of $\alpha, \gamma \in \mathrm{C}^{m}$ and $\langle\beta, \delta\rangle$ the inner product of $\beta, \delta \in \mathbf{E}^{n}$.

A vector subspace $L$ of complex Euclidean $m$-space $\mathbf{C}^{m}$ is called totally real if $J(L) \perp L$, where $J$ denotes the complex structure on $\mathbf{C}^{m}$. A submanifold $M$ in $\mathbf{C}^{m}$ is called totally real if each tangent space of $M$ is totally real.

A submanifold $M$ in $\mathbf{C}^{m}$ is called a $C R$-submanifold if there exists on $M$ a differentiable holomorphic distribution $\mathcal{H}$ whose orthogonal complementary distribution $\mathcal{H}^{\perp}$ is a totally real distribution, that is $J \mathcal{H}^{\perp} \subset T^{\perp} M$ (see $[1, \mathbf{2}, \mathbf{3}, \mathbf{4}, \mathbf{5}]$ ). 


\section{SOME NATURAL EXAMPLES OF CONVOLUTION MANIFOLDS}

Let $\mathbf{C}_{*}^{n}$ and $\mathbf{E}_{*}^{m}$ denote $\mathbf{C}^{n}-\{0\}$ and $\mathbf{E}^{m}-\{0\}$, respectively. Let $\left(z_{1}, \ldots, z_{n}\right)$ denote a complex Euclidean coordinate system of $\mathrm{C}^{n}$ and $\left(x_{1}, \ldots, x_{m}\right)$ a Euclidean coordinate system of $\mathbf{E}^{m}$.

The following result shows that the notion of convolution of Riemannian manifolds arises naturally.

PROPOSITION 3.1. For each holomorphic isometric immersion $z:\left(N_{1}, g_{1}\right)$ $\rightarrow \mathrm{C}_{*}^{n}$ and each isometric immersion $x:\left(N_{2}, g_{2}\right) \rightarrow \mathrm{E}_{*}^{m}$, the map

$$
\psi: N_{1} \times N_{2} \rightarrow \mathbf{C}^{n} \otimes \mathbf{E}^{m}=\mathbf{C}^{n m} ;(u, v) \mapsto z(u) \otimes x(v), u \in N_{1}, v \in N_{2},
$$

gives rise to a convolution manifold $N_{1} \star N_{2}$ equipped with

$$
{ }_{\mu} g_{1} *_{\lambda} g_{2}=\mu^{2} g_{1}+\lambda^{2} g_{2}+2 \lambda \mu d \lambda \otimes d \mu,
$$

where $\lambda=|z|=\sqrt{\sum_{j=1}^{h} z_{j} \bar{z}_{j}}$ and $\mu=|x|=\sqrt{\sum_{\alpha=1}^{p} x_{\alpha}^{2}}$.

Proof: For vector fields $X, Y$ tangent to $N_{1}$ and $Z, W$ tangent to $N_{2}$, we have

$$
d \psi(X)=X \psi=X \otimes x, \quad d \psi(Z)=Z \psi=z \otimes Z .
$$

Also, it follows from the definition of the gradient of $\mu=|x|$ that

$$
\mu d \mu(\nabla \mu)=\frac{\left(\sum_{\alpha=1}^{p} x_{\alpha}\left(e_{1} x_{\alpha}\right)\right)^{2}}{|x|}=\langle\nabla \mu, x\rangle,
$$

where $e_{1}$ is a unit vector parallel to gradient of $\mu$. Similarly, we have

$$
\lambda d \lambda(\nabla \lambda)=\langle\nabla \lambda, z\rangle .
$$

From (2.6), (3.3), (3.4) and (3.5), we obtain Proposition 3.1.

Proposition 3.1 provides many examples of convolution manifolds.

REMARK 3.1. If $x\left(N_{2}\right)$ is contained in the unit hypersphere of $\mathbf{C}^{m}$ centred at the origin, then the convolution $g_{1} * g_{2}$ on the convolution manifold $N_{1} \star N_{2}$ given in Proposition 3.1 is nothing but the warped product metric $g_{1}+|z|^{2} g_{2}$.

\section{GeOMETRY OF $\mathrm{C}_{*}^{h} \circledast \mathrm{E}_{*}^{p}$}

In this section we study the geometry of the tensor product $\mathrm{C}^{h} \otimes \mathrm{E}^{p}$ by applying the notion of convolution.

Assume that $z: \mathbf{C}_{*}^{h} \rightarrow \mathbf{C}^{h}$ and $x: \mathbf{E}_{*}^{p} \rightarrow \mathbf{E}^{p}$ are the inclusion maps. Let $\psi_{z, x}=z \otimes x$ be the map from $\mathbf{C}_{*}^{h} \times \mathrm{E}_{*}^{p}$ into $\mathbf{C}^{h p}$ defined by

$$
\psi_{z, x}=z \otimes x=\left(z_{1} x_{1}, \ldots, z_{1} x_{p}, \ldots, z_{h} x_{1}, \ldots, z_{h} x_{p}\right)
$$


for $z=\left(z_{1}, \ldots, z_{h}\right) \in \mathbf{C}_{*}^{h}$ and $x=\left(x_{1}, \ldots, x_{p}\right) \in \mathbf{E}_{*}^{p}$.

If we put $z_{j}=u_{j}+i v_{j}, i=\sqrt{-1}$, and $\frac{\partial}{\partial z_{j}}=(1 / 2)\left(\frac{\partial}{\partial u_{j}}-i \frac{\partial}{\partial v_{j}}\right)$ for $j=1, \ldots, h$, then we obtain from (4.1) that

$$
d \psi_{z, x}\left(\sum_{j=1}^{h} z_{j} \frac{\partial}{\partial z_{j}}\right)=d \psi_{z, x}\left(\sum_{\alpha=1}^{h} x_{\alpha} \frac{\partial}{\partial x_{\alpha}}\right) .
$$

Notice that $\sum_{j=1}^{h} z_{j} \frac{\partial}{\partial z_{j}}$ and $\sum_{\alpha=1}^{h} x_{\alpha} \frac{\partial}{\partial x_{\alpha}}$ are nothing but the position vectors of $\mathbf{C}_{*}^{h}$ and $\mathbf{E}_{*}^{p}$ in $\mathbf{C}^{h}$ and $\mathbf{E}^{p}$, respectively. Equation (4.2) implies that the gradient of $|z|=\sqrt{\sum_{j=1}^{h} z_{j} \bar{z}_{j}}$ and of $|x|=\sqrt{\sum_{\alpha+1}^{p} x_{\alpha}^{2}}$ are mapped to the same vector field under $\psi_{z, x}$.

From (4.1) and (4.2) it follow that $d \psi_{z, x}$ has constant rank $2 h+p-1$. Hence $\psi_{z, x}\left(\mathbf{C}_{*}^{h} \times \mathbf{E}_{*}^{p}\right)$ gives rise to a $(2 h+p-1)$-manifold, denoted by $\mathbf{C}_{*}^{h} \circledast \mathbf{E}_{*}^{p}$, which is equipped with a Riemannian metric induced from the canonical metric on $\mathbf{C}^{h} \otimes \mathbf{E}^{p}$ via $\psi_{z, x}$. From (4.1) we can verify that $\mathbf{C}_{*}^{h} \circledast \mathbf{E}_{*}^{p}$ is isometric to the warped product $\mathbf{C}_{*}^{h} \times S^{p-1}$ with the warped product metric $g=g_{1}+\rho_{1} g_{0}$, where $\rho_{1}$ is the length of the position function of $\mathbf{C}_{*}^{h}$ and $g_{0}$ the metric of the unit hypersphere $S^{p-1}$.

If we denote the vector field of (4.2) by $V$, then $V$ is a tangent vector field of $\mathbf{C}_{*}^{h} \circledast \mathbf{E}_{*}^{p}$ with length $|x||z|$.

Let

$$
\pi: \mathbf{C}_{*}^{h} \times \mathbf{E}_{*}^{p} \rightarrow \mathbf{C}_{*}^{h} \circledast \mathbf{E}_{*}^{p}
$$

denote the projection: $\pi(u, v)=\psi_{z, x}(u, v)=\{u\} \circledast\{v\} \in \mathbf{C}_{*}^{h} \circledast \mathbf{E}_{*}^{p}$. It is easy to see that, for each $u \in \mathbf{C}_{*}^{h}$ and $v \in \mathbf{E}_{*}^{p}, \mathbf{C}_{*}^{h} \circledast\{v\}=: \psi_{z, x}\left(\mathbf{C}_{*}^{h} \times\{v\}\right)$ is a complex submanifold of complex dimension $h$ and $\{u\} \circledast \mathbf{E}_{*}^{p}=: \psi_{z, x}\left(\{u\} \times \mathbf{E}_{*}^{p}\right)$ is a totally real submanifold of dimension $p$ in $\mathbf{C}^{h p}$.

On $\mathrm{C}_{*}^{h} \times \mathrm{E}_{*}^{p}$, if we put

$$
\begin{array}{lll}
\mathcal{D}=T\left(\mathbf{C}_{*}^{h}\right), & \mathcal{D}^{\perp}=\left\{Z \in T\left(\mathbf{E}_{*}^{p}\right): Z \mu=0\right\}, & \mu=|x|, \\
\mathcal{F}=T\left(\mathbf{E}_{*}^{p}\right), & \mathcal{F}^{\perp}=\left\{X \in T\left(\mathbf{C}_{*}^{h}\right): X \lambda=0\right\}, & \lambda=|z|,
\end{array}
$$

then $\mathcal{D}, \mathcal{D}^{\perp}, \mathcal{F}$ and $\mathcal{F}^{\perp}$ can be regarded as distributions on $\mathbf{C}_{*}^{h} \times \mathbf{E}_{*}^{p}$ in a natural way. Moreover, if we put

$$
\widehat{\mathcal{D}}=d \pi(\mathcal{D}), \quad \widehat{\mathcal{D}}^{\perp}=d \pi\left(\mathcal{D}^{\perp}\right), \widehat{\mathcal{F}}=d \pi(\mathcal{F}), \quad \widehat{\mathcal{F}}^{\perp}=d \pi\left(\mathcal{F}^{\perp}\right),
$$

we have the following orthogonal decompositions of the tangent bundle of $\mathbf{C}_{*}^{h} \circledast \mathbf{E}_{*}^{p}$ :

$$
T\left(\mathbf{C}_{*}^{h} \circledast \mathbf{E}_{*}^{p}\right)=\widehat{\mathcal{D}} \oplus \widehat{\mathcal{D}}^{\perp}=\widehat{\mathcal{F}}^{\perp} \oplus \widehat{\mathcal{F}}
$$


Since the Riemannian metric on $\mathbf{C}_{*}^{h} \circledast \mathbf{E}_{*}^{p}$ is induced from the convolution:

$$
{ }_{h} g_{1} *_{f} g_{2}=\mu^{2} g_{1}+\lambda^{2} g_{2}+2 \lambda \mu d \lambda \otimes d \mu, \quad \lambda=|z|, \quad \mu=|x|,
$$

the distributions $\widehat{\mathcal{D}}$ and $\widehat{\mathcal{D}}^{\perp}$ can be regarded as the tangent and normal bundles of $\mathbf{C}^{h} \circledast$ $\{v\}, v \in \mathbf{E}_{*}^{p}$, in $\mathbf{C}_{*}^{h} \circledast \mathbf{E}_{*}^{p}$, respectively. Similarly, the distributions $\widehat{\mathcal{F}}$ and $\widehat{\mathcal{F}}^{\perp}$ can be regarded as the tangent and normal bundles of $\{u\} \circledast \mathbf{E}_{*}^{p}, u \in \mathbf{C}_{*}^{h}$, in $\mathbf{C}_{*}^{h} \circledast \mathbf{E}_{*}^{p}$, respectively.

We give the following two lemmas for later use.

LEMma 4.1. Let $\nabla$ denote the Riemannian connection of $\mathrm{C}_{*}^{h} \circledast \mathrm{E}_{*}^{p}$. Then, for any vector fields $X$ in $\widehat{\mathcal{F}}^{\perp}$ and $Z$ in $\widehat{\mathcal{D}}^{\perp}$, we have

(a) $\nabla_{X} Z=\nabla_{Z} X=0$

(b) $\nabla_{V} Z=\nabla_{Z} V=Z$, and

(c) $\nabla_{V} X=\nabla_{X} V=X$,

where $V$ is the vector field given by (4.2).

Proof: For each vector field $X$ in $\widehat{\mathcal{F}}^{\perp}$ and $Z$ in $\widehat{\mathcal{D}}^{\perp}$, there exist vector fields $\check{X}$ in $\mathcal{F}^{\perp}$ and $\breve{Z}$ in $\mathcal{D}^{\perp}$ such that $d \pi(\breve{X})=X$ and $d \pi(\breve{Z})=Z$. From (4.1), we have

$$
\breve{X} \breve{Z} \psi_{z, x}=\breve{X} \otimes \breve{Z} .
$$

Since $\breve{X} \lambda=\breve{Z} \mu=0, \lambda=|z|, \mu=|x|$, the vector field $\breve{X} \otimes \breve{Z}$ is perpendicular to $d \psi_{z, x}\left(\frac{\partial}{\partial z_{1}}\right), \ldots, d \psi_{z, x}\left(\frac{\partial}{\partial z_{h}}\right), d \psi_{z, x}\left(\frac{\partial}{\partial x_{1}}\right), \ldots, d \psi_{z, x}\left(\frac{\partial}{\partial x_{p}}\right)$. Thus, for any vector fields $X$ in $\widehat{\mathcal{F}}^{\perp}$ and $Z$ in $\widehat{\mathcal{D}}^{\perp}$, we have (a).

Let $\breve{V}=z=\sum_{j=1}^{h} z_{j} \partial / \partial z_{j} \in T\left(\mathbf{C}^{h}\right)$ and let $\breve{Z}$ be any vector in $\mathcal{D}^{\perp}$. Then (4.1) implies

$$
\breve{Z} \breve{V} \psi_{z, x}=\breve{V} \otimes \breve{Z} .
$$

Since $z \otimes \breve{Z}=\breve{V} \otimes \breve{Z}$ is tangent to $\mathbf{C}_{*}^{h} \circledast \mathbf{E}_{*}^{p},(4.9)$ implies

$$
\nabla_{V} Z=\breve{V} \otimes \breve{Z} .
$$

On the other hand, we also have $Z=d \psi_{z, x}(\breve{Z})=\breve{Z} \psi_{z, x}=\breve{V} \otimes \breve{Z}$ from (4.1). By comparing this with (4.10), we obtain (b).

Similarly, let $\widetilde{V}=x=\sum_{\alpha=1}^{p} x_{\alpha} \partial / \partial x_{\alpha}$ and let $\check{X}$ be any vector field in $\mathcal{F}^{\perp}$, we have

$$
\breve{X} \widetilde{V} \psi_{z, x}=\breve{X} \otimes x .
$$

On the other hand, we also have $X=d \psi_{z, x}(\breve{X})=\breve{X} \psi_{z, x}=\breve{X} \otimes x$. Comparing this with (4.11) gives (c). 
Lemma 4.2. For each $u \in \mathbf{C}_{*}^{h}$ and each $v \in \mathbf{E}_{*}^{p},\{u\} \circledast \mathbf{E}_{*}^{p}$ and $\mathbf{C}_{*}^{h} \circledast\{v\}$ are totally geodesic submanifolds of $\mathbf{C}_{*}^{h} \circledast \mathbf{E}_{*}^{p}$.

Proof: First, we recall that the tangent and normal bundles of $\mathbf{C}_{*}^{h} \circledast\{v\}$ in $\mathbf{C}_{*}^{h} \circledast \mathbf{E}_{*}^{p}$ are given respectively by $\widehat{\mathcal{D}}$ and $\widehat{\mathcal{D}}^{\perp}$. Since the distribution $\widehat{\mathcal{D}}$ is spanned by $\widehat{\mathcal{F}}^{\perp}$ and $V$, statements (a) and (b) of Lemma 4.1 and the formula of Weingarten imply that $\mathbf{C}_{*}^{h} \circledast\{v\}$ is totally geodesic in $\mathrm{C}_{*}^{h} \circledast \mathrm{E}_{*}^{p}$.

Similarly, from statements (b) and (c) of Lemma 4.1 and the formula of Weingarten, we conclude that each $\{u\} \circledast \mathbf{E}_{*}^{p}$ is a totally geodesic submanifold of $\mathbf{C}_{*}^{h} \circledast \mathbf{E}_{*}^{p}$.

\section{Classification of natural $C R$-immersions of $\mathbf{C}_{*}^{h} \circledast \mathbf{E}_{*}^{p}$}

Since there is a canonical holomorphic distribution $\widehat{\mathcal{D}}$ on $\mathrm{C}_{*}^{h} \circledast \mathrm{E}_{*}^{p}$, we call an isometric immersion $\psi: U \rightarrow \mathbf{C}^{\dot{m}}$ of an open portion $U$ of $\mathbf{C}_{*}^{h} \circledast \mathbf{E}_{*}^{p}$ into $\mathbf{C}^{m}$ a natural $C R$ immersion if $\psi$ carries $\widehat{\mathcal{D}}$ into a holomorphic distribution in $\mathbf{C}^{n}$ and carries the orthogonal complementary distribution $\widehat{\mathcal{D}}^{\perp}$ of $\widehat{\mathcal{D}}$ into a totally real distribution. Clearly, (4.1) defines a natural $C R$-immersion of $\mathrm{C}_{*}^{h} \circledast \mathbf{E}_{*}^{p}$ into $\mathrm{C}^{h p}$.

Theorem 5.1. Let $\phi: U \rightarrow \mathrm{C}^{m}$ be a natural $C R$-immersion from an open portion $U$ of $\mathbf{C}_{*}^{h} \circledast \mathbf{E}_{*}^{p}$ into $\mathbf{C}^{m}$. Then, up to rigid motions of $\mathbf{C}^{m}, \tilde{\phi}=\phi \circ \pi$ is given by

$$
\widetilde{\phi}(z, x)=\sum_{j=1}^{h} A^{j}\left(x_{1}, \ldots, x_{p}\right) z_{j},
$$

where $A^{1}, \ldots, A^{h}$ are mutually orthogonal vector functions of length $|x|$ which span a totally real subspace of $\mathbf{C}^{m}$ at each point $x=\left(x_{1}, \ldots, x_{p}\right)$ with $\pi(z, x) \in U$. Moreover, $A^{1}, \ldots, A^{h}$ satisfy

$$
\begin{aligned}
\left\langle A^{j}, A_{x_{\alpha}}^{k}\right\rangle & =x_{\alpha} \delta_{j k},\left\langle A_{x_{\alpha}}^{j}, A_{x_{\beta}}^{k}\right\rangle=\delta_{j k} \delta_{\alpha \beta}, \\
\left\langle A^{j}, i A_{x_{\alpha}}^{k}\right\rangle & =\left\langle A_{x_{\alpha}}^{j}, i A_{x_{\beta}}^{k}\right\rangle=0
\end{aligned}
$$

for $j, k=1 \ldots, h ; \alpha, \beta=1, \ldots, p$, where $A_{x_{\alpha}}^{j}=\partial A^{j} / \partial x_{\alpha}$.

Conversely, if $A^{1}, \ldots, A^{h}$ are $h$ mutually orthogonal $\mathbf{E}^{m}$-valued functions of length $|x|$ satisfying (5.2), then (5.1) defines a natural $C R$-immersion of $\mathbf{C}_{*}^{h} \circledast \mathbf{E}_{*}^{p}$ into $\mathbf{C}^{m}$.

PROOF: Suppose that $\phi: U \rightarrow \mathrm{C}^{m}$ is a natural $C R$-immersion from an open portion of $\mathrm{C}_{*}^{h} \circledast \mathrm{E}_{*}^{p}$ into $\mathrm{C}^{m}$. For each $v \in \mathbf{E}_{*}^{p}$, Lemma 4.2 implies that $\mathbf{C}_{*}^{h} \circledast\{v\}$ is a totally geodesic submanifold of $\mathbf{C}_{*}^{h} \circledast \mathbf{E}_{*}^{p}$.

Because the restriction of $\phi$ to $U \cap\left(\mathbf{C}_{*}^{h} \circledast\{v\}\right)$ is a holomorphically isometric immersion of $U \cap\left(\mathbf{C}_{*}^{h} \circledast\{v\}\right)$ into $\mathbf{C}^{m}$ and $\mathbf{C}_{*}^{h} \circledast\{v\}$ is a flat Kaehler manifold, the equation of Gauss implies that the restriction of $\phi$ to $U \cap\left(\mathbf{C}_{*}^{h} \circledast\{v\}\right)$ is a totally geodesic holomorphic immersion. Thus, $\phi$ immerses $U \cap\left(\mathbf{C}_{*}^{h} \circledast\{v\}\right)$ into a complex $h$-plane in 
$\mathbf{C}^{m}$. Consequently, $\tilde{\phi}$ immerses $\pi^{-1}(U) \cap\left(\mathbf{C}_{*}^{h} \times\{v\}\right)$ into a complex $h$-plane in $\mathbf{C}^{m}$. Hence, we have

$$
\widetilde{\phi}_{z_{j} z_{k}}=\tilde{\phi}_{z_{j} \bar{z}_{k}}=\tilde{\phi}_{\bar{z}_{j} \bar{z}_{k}}=0, \quad 1 \leqslant j, k \leqslant h .
$$

Solving (5.3) yields

$$
\widetilde{\phi}(z, x)=\sum_{j=1}^{h} A^{j}\left(x_{1}, \ldots, x_{p}\right) z_{j}+B\left(x_{1}, \ldots, x_{p}\right)
$$

for some $\mathrm{C}^{m}$-valued functions $A^{1}, \ldots, A^{h}$ and $B$. From (5.4) we find

$$
\widetilde{\phi}_{x_{\alpha}}=\sum_{j=1}^{h} A_{x_{\alpha}}^{j} z_{j}+B_{x_{\alpha}}, \quad \alpha=1, \ldots, p .
$$

Thus, by (3.15) and (5.5), we obtain

$$
\sum_{j=1}^{h} z_{j} \bar{z}_{j}=\left\langle\sum_{j=1}^{h} A_{x_{\alpha}}^{j} z_{j}, \sum_{k=1}^{h} A_{x_{\alpha}}^{k} z_{k}\right\rangle+2\left\langle\sum_{j=1}^{h} A_{x_{\alpha}}^{j} z_{j}, B_{x_{\alpha}}\right\rangle+\left\langle B_{x_{\alpha}}, B_{x_{\alpha}}\right\rangle .
$$

Condition (5.6) implies $B_{x_{1}}=\cdots=B_{x_{p}}=0$. Hence, $B$ is a constant vector in $\mathrm{C}^{m}$. Without loss of generality, we may choose $B=0$ by applying a suitable translation on $\mathrm{C}^{m}$ if necessary. Hence, $(5.4)$ reduces to

$$
\widetilde{\phi}(z, x)=\sum_{j=1}^{h} A^{j}\left(x_{1}, \ldots, x_{p}\right) z_{j}
$$

From (5.7), we obtain

$$
\widetilde{\phi}_{u_{j}}=A^{j}, \quad \widetilde{\phi}_{v_{j}}=i A^{j}, \quad \widetilde{\phi}_{x_{\alpha}}=\sum_{j=1}^{h} A_{x_{\alpha}}^{j} z_{j}
$$

for $j=1, \ldots, h ; \alpha=1, \ldots, p$, where $z_{j}=u_{j}+i v_{j}$. From (3.15) and (5.8), we find

$$
\begin{aligned}
|x|^{2} \delta_{j k} & =\left\langle\widetilde{\phi}_{u_{j}}, \tilde{\phi}_{u_{k}}\right\rangle=\left\langle A^{j}, A^{k}\right\rangle,\left\langle A^{j}, i A^{k}\right\rangle=\left\langle\tilde{\phi}_{u_{j}}, \tilde{\phi}_{v_{k}}\right\rangle=0, \\
u_{j} x_{\alpha} & =\left\langle\tilde{\phi}_{u_{j}}, \tilde{\phi}_{x_{\alpha}}\right\rangle=\left\langle A^{j}, \sum_{k=1}^{h} A_{x_{\alpha}}^{k} z_{k}\right\rangle, \\
v_{j} x_{\alpha}=\left\langle\tilde{\phi}_{v_{j}}, \tilde{\phi}_{x_{\alpha}}\right\rangle & =\left\langle i A^{j}, \sum_{k=1}^{h} A_{x_{\alpha}}^{k} z_{k}\right\rangle, \\
|z|^{2} \delta_{\alpha \beta} & =\left\langle\tilde{\phi}_{x_{\alpha}}, \tilde{\phi}_{x_{\beta}}\right\rangle=\left\langle\sum_{j=1}^{h} A_{x_{\alpha}}^{j} z_{j}, \sum_{k=1}^{h} A_{x_{\beta}}^{k} z_{k}\right\rangle
\end{aligned}
$$


for $j, k=1, \ldots, h ; \alpha, \beta=1, \ldots, p$.

The first equation of (5.9) implies that $A^{1}, \ldots, A^{h}$ are $h$ mutually orthogonal vector functions of length $|x|$. From equations (5.9), (5.10) and (5.11) we have

$$
\left\langle A^{j}, i A^{k}\right\rangle=0, \quad\left\langle A^{j}, A_{x_{\alpha}}^{k}\right\rangle=x_{\alpha} \delta_{j k}, \quad\left\langle A^{j}, i A_{x_{\alpha}}^{k}\right\rangle=0
$$

for $j, k=1, \ldots, h ; \alpha, \beta=1, \ldots, p$.

By comparing the coefficients of $u_{j} u_{k}$ from (5.12), we find

$$
\left\langle A_{x_{\alpha}}^{j}, A_{x_{\beta}}^{k}\right\rangle+\left\langle A_{x_{\beta}}^{j}, A_{x_{\alpha}}^{k}\right\rangle=2 \delta_{j k} \delta_{\alpha \beta}
$$

for $j, k=1, \ldots, h ; \alpha, \beta=1, \ldots, p$. If $j=k,(5.14)$ implies

$$
\left\langle A_{x_{\alpha}}^{j}, A_{x_{\beta}}^{j}\right\rangle=\delta_{\alpha \beta} .
$$

If $j \neq k$, then, by taking partial derivative of $\left\langle A^{j}, A_{x_{\alpha}}^{k}\right\rangle=0$ with respect to $x_{\beta}$, we obtain

$$
\left\langle A_{x_{\alpha}}^{j}, A_{x_{\beta}}^{k}\right\rangle=-\left\langle A^{j}, A_{x_{\beta} x_{\alpha}}^{k}\right\rangle=\left\langle A_{x_{\beta}}^{j}, A_{x_{\alpha}}^{k}\right\rangle, \quad j \neq k .
$$

Combining this with (5.14) yields $\left\langle A_{x_{\alpha}}^{j}, A_{x_{\beta}}^{k}\right\rangle=0$ for $j \neq k$. Hence, we have the second equation of (5.2). By comparing the coefficients of $u_{j} v_{k}$ from (5.12), we also find

$$
\left\langle A_{x_{\alpha}}^{j}, i A_{x_{\beta}}^{k}\right\rangle+\left\langle i A_{x_{\alpha}}^{k}, A_{x_{\beta}}^{j}\right\rangle=0
$$

for $1 \leqslant j \neq k \leqslant h$ and $1 \leqslant \alpha, \beta \leqslant p$.

On the other hand, by applying $\left\langle\widetilde{\phi}_{x_{\alpha}}, J \tilde{\phi}_{x_{\beta}}\right\rangle=0$ and (5.8), we find

$$
\left\langle\sum_{j=1}^{h} A_{x_{\alpha}}^{j} z_{j}, \sum_{k=1}^{h} i A_{x_{\beta}}^{k} z_{k}\right\rangle=0 .
$$

Comparing the coefficients of $u_{j} u_{k}$ from (5.18) yields

$$
\left\langle A_{x_{\alpha}}^{j}, i A_{x_{\beta}}^{k}\right\rangle+\left\langle A_{x_{\alpha}}^{k}, i A_{x_{\beta}}^{j}\right\rangle=0
$$

for $j, k=1, \ldots, h ; \alpha, \beta=1, \ldots, p$.

If $j=k,(5.19)$ implies $\left\langle A_{x_{\alpha}}^{j}, i A_{x_{\beta}}^{j}\right\rangle=0$. If $j \neq k$, then, by combining (5.17) and (5.19), we obtain $\left\langle A_{x_{\alpha}}^{j}, i A_{x_{\beta}}^{k}\right\rangle=0$. Therefore, we have $\left\langle A_{x_{\alpha}}^{j}, i A_{x_{\beta}}^{k}\right\rangle=0$ for $j, k=1, \ldots, h$; $\alpha, \beta=1, \ldots, p$. Consequently, we have (5.2). From (5.2) we know that $A^{1}, \ldots, A^{h}$ span a totally real subspace of $\mathbf{C}^{m}$ at each point $x=\left(x_{1}, \ldots, x_{p}\right)$ with $\pi(z, x) \in U$.

Statement (b) can be proved by straightforward computation.

EXAMPLE 5.1. Let $D$ be an open portion of Euclidean $p$-space $\mathbf{E}^{p}$ which does not contain the origin of $\mathrm{E}^{p}$. If $A: D \rightarrow \mathbf{E}^{m}$ is an isometric immersion of the flat space 
$D$ into $\mathbf{E}^{m}$ satisfying $|A|=|x|$ at $x=\left(x_{1}, \ldots, x_{p}\right) \in D$, then (5.2) holds automatically. Hence, by Theorem 5.1, we know that

$$
\widetilde{\phi}(z, x)=A\left(x_{1}, \ldots, x_{p}\right) z
$$

defines a natural $C R$-immersion from $\mathbf{C}_{*}^{1} \circledast D$ into $\mathbf{C}^{m}$. In particular, if $\gamma(s)$ $=\left(\gamma_{1}(s), \ldots, \gamma_{m-p+1}(s)\right)$ is a unit speed curve satisfying

$$
|\gamma(s)|^{2}=\sum_{j=1}^{m+p-1} \gamma_{j}^{2}(s)=s^{2},
$$

then $A=\left(\gamma_{1}\left(x_{1}\right), \ldots, \gamma_{m-p+1}\left(x_{1}\right), x_{2}, \ldots, x_{p}\right)$ defines an isometric immersion of an open portion $D$ of $\mathrm{E}_{*}^{p}$ into $\mathrm{E}^{m}$ satisfying $|A|=|x|$. Thus,

$$
\widetilde{\phi}(z, x)=A\left(x_{1}, \ldots, x_{p}\right) z, \quad A=\left(\gamma_{1}\left(x_{1}\right), \ldots, \gamma_{m-p+1}\left(x_{1}\right), x_{2}, \ldots, x_{p}\right)
$$

defines a natural $C R$-immersion from $\mathbf{C}_{*}^{1} \circledast D$ into $\mathbf{C}^{m}$.

REMARK 5.1. When $m+p-1=2$, then $\gamma_{1}=a s, \gamma_{2}=b s, a^{2}+b^{2}=1$, are the only functions satisfying (5.21). However, if $m+p-1 \geqslant 3$, then there are many unit speed curves $\gamma$, other than lines, which satisfy (5.21).

EXAMPle 5.2. Suppose that $D$ is an open portion of Euclidean p-space which does not contain the origin. Let $A^{j}: D \rightarrow \mathrm{E}^{m_{j}}, j=1, \ldots, h$, be isometric immersions of the flat space $D$ into $\mathbf{E}^{m_{j}}$ satisfying $\left|A^{1}\right|=\cdots=\left|A^{h}\right|=|x|$ on $D$, then

$$
\psi(z, x)=\left(A^{1}\left(x_{1}, \ldots, x_{p}\right) z_{1}, \ldots, A^{h}\left(x_{1}, \ldots, x_{p}\right) z_{h}\right)
$$

defines a natural $C R$-immersion from $\mathbf{C}_{*}^{h} \circledast D$ into $\mathbf{C}^{m}, m=m_{1}+\cdots+m_{h}$.

\section{TWO GEOMETRIC CHARACTERISATIONS OF $\psi_{z, x}$}

The following result provides a simple geometric characterisation of $\psi_{z, x}=z \otimes x$.

THEOREM 6.1. If $\phi: U \rightarrow \mathbf{C}^{m}$ is a natural $C R$-immersion of an open portion $U$ of $\mathrm{C}_{*}^{h} \circledast \mathrm{E}_{*}^{p}$ into $\mathrm{C}^{m}$, then we have:

(1) The squared norm of the second fundamental form $\sigma$ of $\phi$ satisfies

$$
\|\sigma\|^{2} \geqslant \frac{(2 h-1)(p-1)}{|x|^{2}|z|^{2}} .
$$

(2) The equality sign of (6.1) holds identically if and only if, up to rigid motions of $\mathrm{C}^{m}$, the composition $\widetilde{\phi}=\phi \circ \pi$ is given by

$$
\widetilde{\phi}(z, x)=\left(z_{1} x_{1}, \ldots, z_{1} x_{p}, \ldots, z_{h} x_{1}, \ldots, z_{h} x_{p}, 0, \ldots, 0\right)
$$


where $\pi: \mathbf{C}_{*}^{h} \times \mathbf{E}_{*}^{p} \rightarrow \mathbf{C}_{*}^{h} \circledast \mathrm{E}_{*}^{p}$ is the projection and $z=\left(z_{1}, \ldots, z_{h}\right)$ and $x=\left(x_{1}, \ldots, x_{p}\right)$ are natural coordinate systems of $\mathbf{C}_{*}^{h}$ and $\mathbf{E}_{*}^{p}$, respectively.

We need some lemmas.

LEMMA 6.1. If $\phi: U \rightarrow \mathbf{C}^{m}$ is a natural $C R$-immersion from an open portion of $\mathbf{C}_{*}^{h} \circledast \mathbf{E}_{*}^{p}$ into $\mathbf{C}^{m}$, then, for any vector fields $X$ in $\widehat{\mathcal{D}}$ with $\langle X, V\rangle=\langle X, J V\rangle=0$ and for $Z, W$ in $\widehat{\mathcal{D}}^{\perp}$, we have

$$
\begin{aligned}
\langle\sigma(X, Z), J W\rangle & =0, \\
\langle\sigma(J V, Z), J W\rangle & =\langle Z, W\rangle, \\
\langle\sigma(V, Z), J W\rangle & =0 .
\end{aligned}
$$

Proof: Suppose $Z, W$ are vector fields in $\widehat{\mathcal{D}}^{\perp}$. Then we have

$$
J \nabla_{Z} W+J \sigma(Z, W)=-A_{J W} Z+D_{Z} J W .
$$

If $X$ is a vector field in $\widehat{\mathcal{D}}$ with $\langle X, V\rangle=\langle X, J V\rangle=0$, then both $X$ and $J X$ belong to $\widehat{\mathcal{F}}^{\perp}$. Thus, by $(6.6)$, we have

$$
\langle\sigma(J X, Z), J W\rangle=\left\langle A_{J W} Z, J X\right\rangle=-\left\langle\nabla_{Z} W, X\right\rangle=\left\langle\nabla_{Z} X, W\right\rangle=0
$$

for $X$ in $\mathcal{D}$ and $Z, W$ on $\mathcal{D}^{\perp}$. This proves (6.3).

From (6.6) and statement (b) of Lemma 4.1, we find, for $Z, W$ in $\widehat{\mathcal{D}}^{\perp}$, that

$$
\langle\sigma(J V, Z), J W\rangle=\left\langle A_{J W} Z, J V\right\rangle=-\left\langle\nabla_{Z} W, V\right\rangle=\left\langle W, \nabla_{Z} V\right\rangle=\langle W, Z\rangle
$$

which proves $(6.4)$.

For $Z, W$ in $\widehat{\mathcal{D}}^{\perp}$, we also find from (6.6) and Lemma 4.1 that

$$
\langle\sigma(V, Z), J W\rangle=\left\langle A_{J W} Z, V\right\rangle=\left\langle\nabla_{Z} W, J V\right\rangle=\left\langle W, \nabla_{Z} J V\right\rangle=0,
$$

since $J V \in \widehat{\mathcal{F}}^{\perp}$. This proves (6.5).

LEMMA 6.2. If $\phi: U \rightarrow \mathbf{C}^{m}$ is a natural $C R$-immersion of an open portion of $\mathbf{C}_{*}^{h} \circledast \mathbf{E}_{*}^{p}$ into $\mathbf{C}^{m}$, then, for $X \in \widehat{\mathcal{F}}$ with $\langle X, V\rangle=\langle X, J V\rangle=0$ and $Z \in \widehat{\mathcal{D}}^{\perp}$, the second fundamental form $\sigma$ of $\phi$ satisfies

$$
|\sigma(X, Z)|=\frac{|X||Z|}{|x||z|}
$$

Proof: For $X \in \widehat{\mathcal{D}}$ with $\langle X, V\rangle=\langle X, J V\rangle=0$ and $Z \in \mathcal{D}^{\perp}$, the equation of Codazzi implies

$$
\begin{aligned}
& \left\langle D_{J X} \sigma(X, Z)-\sigma\left(\nabla_{J X} X, Z\right)-\sigma\left(X, \nabla_{J X} Z\right), J Z\right\rangle \\
& \quad=\left\langle D_{X} \sigma(J X, Z)-\sigma\left(\nabla_{X} J X, Z\right)-\sigma\left(J X, \nabla_{X} Z\right), J Z\right\rangle
\end{aligned}
$$


Since $\mathbf{C}_{*}^{h} \circledast\{v\}$ is a totally geodesic submanifold of $\mathbf{C}_{*}^{h} \circledast \mathbf{E}_{*}^{p}$ according to Lemma 4.2 , $\nabla_{X} J X$ and $\nabla_{J X} X$ belong to $\widehat{\mathcal{D}}$. From Lemma 4.1, we also know that $\nabla_{X} Z$ and $\nabla_{J X} Z$ belong to $\widehat{\mathcal{D}}^{\perp}$. Hence, by (6.11), Lemma 4.1, and Lemma 6.1, we get

$$
\begin{aligned}
\left\langle\sigma(X, Z), D_{J X} J Z\right\rangle+\langle & \left\langle\sigma\left(\nabla_{J X} X, Z\right), J Z\right\rangle \\
& =\left\langle\sigma(J X, Z), D_{X} J Z\right\rangle+\left\langle\sigma\left(\nabla_{X} J X, Z\right), J Z\right\rangle .
\end{aligned}
$$

From formulas of Gauss and Weingarten, we have

$$
J \nabla_{X} Z+J \sigma(X, Z)=-A_{J Z} X+D_{X} J Z .
$$

Since $\nabla_{X} Z$ lies in $\widehat{\mathcal{D}}^{\perp},(6.13)$, Lemma 4.1 , and Lemma 6.1 imply

$$
\begin{aligned}
\left\langle\sigma(J X, Z), D_{X} J Z\right\rangle & =\left\langle\sigma(J X, Z), J \nabla_{X} Z\right\rangle+\langle\sigma(J X, Z), J \sigma(X, Z)\rangle \\
& =\langle\sigma(J X, Z), J \sigma(X, Z)\rangle .
\end{aligned}
$$

Let $\nu$ denote the orthogonal complement of $J \widehat{\mathcal{D}}^{\perp}$ in the normal bundle of $\mathrm{C}_{*}^{h} \circledast \mathrm{E}_{*}^{p}$ in $\mathbf{C}^{m}$. Then $\nu$ is invariant under the action of the complex structure $J$ of $\mathbf{C}^{m}$. We denote by $\sigma_{\nu}$ the $\nu$-component of the second fundamental form $\sigma$.

Let $\tilde{\nabla}$ be the Riemannian connection of $\mathbf{C}^{m}$. For each $\xi \in \nu, Y \in \widehat{\mathcal{D}}$, and each tangent vector $U$ of $\mathbf{C}_{*}^{h} \circledast \mathbf{E}_{*}^{p}$, we have

$$
\left\langle A_{\xi}(J Y), U\right\rangle=\langle\sigma(J Y, U), \xi\rangle=\left\langle J \tilde{\nabla}_{U} Y, \xi\right\rangle=-\langle\sigma(Y, U), J \xi\rangle=-\left\langle A_{J \xi} Y, U\right\rangle .
$$

Hence, we get

$$
A_{J \xi} Y=-A_{\xi}(J Y), \quad Y \in \widehat{\mathcal{D}}, \quad \xi \in \nu .
$$

By applying (6.15), we find

$$
\begin{aligned}
\langle\sigma(J X, Z), J \sigma(X, Z)\rangle & =\left\langle\sigma(J X, Z), J \sigma_{\nu}(X, Z)\right\rangle \\
& =\left\langle A_{J \sigma_{\nu}(X, Z)} J X, Z\right\rangle=\left\langle A_{\sigma_{\nu}(X, Z)} X, Z\right\rangle \\
& =\left\langle\sigma(X, Z), \sigma_{\nu}(X, Z)\right\rangle=\left|\sigma_{\nu}(X, Z)\right|^{2} .
\end{aligned}
$$

Combining (6.14) and (6.16), we obtain

$$
\left\langle\sigma(J X, Z), D_{X} J Z\right\rangle=\left|\sigma_{\nu}(X, Z)\right|^{2} .
$$

Replacing $X$ in (6.17) by $J X$ and applying (6.15) yield

$$
\left\langle\sigma(X, Z), D_{J X} J Z\right\rangle=-\left|\sigma_{\nu}(X, Z)\right|^{2} .
$$

On the other hand, by Lemma 4.1 and Lemma 6.1, we have

$$
\begin{aligned}
\left\langle\sigma\left(\nabla_{J X} X, Z\right), J Z\right\rangle & =\frac{\left\langle\nabla_{J X} X, J V\right\rangle}{|x|^{2}|z|^{2}}\langle Z, Z\rangle \\
& =\frac{\left\langle J X, \nabla_{J X} V\right\rangle}{|x|^{2}|z|^{2}}\langle Z, Z\rangle=\frac{|X|^{2}|Z|^{2}}{|x|^{2}|z|^{2}} .
\end{aligned}
$$


Replacing $X$ in (6.19) by $J X$ yields

$$
-\left\langle\sigma\left(\nabla_{X} J X, Z\right), J Z\right\rangle=\frac{|X|^{2}|Z|^{2}}{|x|^{2}|z|^{2}} .
$$

Combining (6.12) and (6.17)-(6.20) gives

$$
\left|\sigma_{\nu}(X, Z)\right|=\frac{|X||Z|}{|x||z|}
$$

for $Z \in \widehat{\mathcal{D}}^{\perp}$ and $X \in \widehat{\mathcal{D}}$ with $\langle X, V\rangle=\langle X, J V\rangle=0$.

On the other hand, from (6.3) of Lemma 6.1, we have $\sigma(X, Z)=\sigma_{\nu}(X, Z)$. Hence, we obtain (6.10) from (6.21). This proves Lemma 6.3.

Now, we return to the proof of Theorem 6.1. First, by applying (6.4) of Lemma 6.1, we have

$$
|\sigma(J V, Z)| \geqslant\langle Z, Z\rangle
$$

with equality holding if and only if $\sigma(J V, Z)=J Z$.

Since $|J V|=|x||z|$, we obtain inequality (6.1) from (6.21) and (6.22).

Suppose that the equality sign of (6.1) holds. Then, by Lemma 6.2, we have

$$
\sigma(\widehat{\mathcal{D}}, \widehat{\mathcal{D}})=0, \quad \sigma(\widehat{\mathcal{F}}, \widehat{\mathcal{F}})=0
$$

for $Z \in \widehat{\mathcal{D}}^{\perp}$ and $X \in \widehat{\mathcal{D}}$ with $\langle X, V\rangle=\langle X, J V\rangle=0$.

Since $\phi: U \rightarrow \mathbf{C}^{m}$ is a natural $C R$-immersion from an open portion of $\mathbf{C}_{*}^{h} \circledast \mathbf{E}_{*}^{p}$ into $\mathbf{C}^{m}$, Theorem 5.1 implies that, up to rigid motions of $\mathbf{C}^{m}$, the composition $\tilde{\phi}=\phi \circ \pi$ is given by

$$
\widetilde{\phi}(z, x)=\sum_{j=1}^{h} A^{j}\left(x_{1}, \ldots, x_{p}\right) z_{j},
$$

where $A^{1}\left(x_{1}, \ldots, x_{p}\right), \ldots, A^{h}\left(x_{1}, \ldots, x_{p}\right)$ are orthogonal vector functions of length $|x|$. On the other hand, from Lemma 4.2 and the second equation of (6.23), we know that, for each $u \in \mathbf{C}_{*}^{h}, \phi$ immerses $U \cap\left(\{u\} \circledast \mathbf{E}_{*}^{p}\right)$ into a totally real $p$-plane in $\mathbf{C}^{m}$. Hence, $\tilde{\phi}$ carries $\pi^{-1}(U) \cap\left(\{u\} \times \mathbf{E}_{*}^{p}\right)$ into a totally real $p$-plane. Therefore, by applying formula of Gauss, we obtain

$$
\widetilde{\phi}_{x_{\alpha} x_{\beta}}=0, \quad \alpha, \beta=1, \ldots, p .
$$

Hence, after solving (6.25), we obtain from (6.24) that

$$
\tilde{\phi}(z, x)=\sum_{j=1}^{h} \sum_{\alpha=1}^{p} c_{\alpha}^{j} x_{\alpha} z_{j}+\sum_{j=1}^{h} b^{j} z_{j}
$$


for some constant vectors $c_{\alpha}^{j}$ and $b^{j}, \alpha=1, \ldots, p ; j=1, \ldots, h$.

Equation (6.26) yields

$$
\tilde{\phi}_{z_{j}}=\sum_{\alpha=1}^{p} c_{\alpha}^{j} x_{\alpha}+b^{j}, \quad \tilde{\phi}_{x_{\alpha}}=\sum_{j=1}^{h} c_{\alpha}^{j} z_{j}
$$

for $j, k=1 \ldots, h ; \alpha, \beta=1, \ldots, p$. Hence, by applying (3.15) and (6.27), we find

$$
|x|^{2}=\left\langle\widetilde{\phi}_{z_{j}}, \tilde{\phi}_{z_{k}}\right\rangle=\left\langle\sum_{\alpha=1}^{p} c_{\alpha}^{j} x_{\alpha}+b^{j}, \sum_{\alpha=1}^{p} c_{\alpha}^{j} x_{\alpha}+b^{j}\right\rangle
$$

which implies $b^{1}=\cdots=b^{h}=0$. Thus, (6.26) becomes

$$
\widetilde{\phi}(z, x)=\sum_{j=1}^{h} A^{j}\left(x_{1}, \ldots, x_{p}\right) z_{j}, \quad A^{j}=\sum_{\alpha=1}^{p} c_{\alpha}^{j} x_{\alpha},
$$

which gives

$$
A_{x_{\alpha}}^{j}=c_{\alpha}^{j}
$$

On the other hand, Theorem 5.1 implies that $A^{1}, \ldots, A^{h}$ satisfy

$$
\left\langle A_{x_{\alpha}}^{j}, A_{x_{\beta}}^{k}\right\rangle=\delta_{j k} \delta_{\alpha \beta}, \quad\left\langle A_{x_{\alpha}}^{j}, i A_{x_{\beta}}^{k}\right\rangle=0
$$

for $j, k=1 \ldots, h ; \alpha, \beta=1, \ldots, p$. Combining (6.30) and (6.31) give

$$
\left\langle c_{\beta}^{j}, c_{\alpha}^{k}\right\rangle=\delta_{j k} \delta_{\alpha \beta}, \quad\left\langle c_{\beta}^{j}, i c_{\alpha}^{k}\right\rangle=0
$$

for $j, k=1 \ldots, h ; \alpha, \beta=1, \ldots, p$. Hence $\left\{c_{\alpha}^{j}, j=1, \ldots, h ; \alpha=1, \ldots, p\right\}$ is an orthonormal set which spans a totally real $h p$-subspace of $\mathbf{C}^{\mathbf{m}}$. Without loss of generality, we may choose the complex coordinates $z_{1}, \ldots, z_{m}$ on $\mathbf{C}^{m}$ such that

$$
\begin{aligned}
& c_{1}^{1}=(1,0, \ldots, 0), \ldots, \\
& c_{p}^{1}=(0, \ldots, 0,1,0, \ldots, 0), \quad(1 \text { appears } p \text {-th place }), \ldots, \\
& c_{1}^{h}=(0, \ldots, 0,1,0, \ldots, 0), \quad(1 \text { appears }(h-1) p+1 \text {-th place }), \ldots, \\
& c_{p}^{h}=(0, \ldots, 0,1,0, \ldots, 0) \quad(1 \text { appears } h p \text {-th place })
\end{aligned}
$$

Combining (6.29) and (6.33) gives (6.2).

Conversely, it is straightforward to verify that (6.2) defines a natural $C R$-immersion of $\mathrm{C}_{*}^{h} \circledast \mathbf{E}_{*}^{p}$ into $\mathbf{C}^{m}$ whose second fundamental form satisfies the equality case of (6.1).

The following theorem provides another simple geometric characterisation of $\psi_{z, x}$ $=z \otimes x$. 
TheOREM 6.2. Let $\phi: U \rightarrow \mathbf{C}^{m}$ be a natural $C R$-immersion from an open portion $U$ of $\mathrm{C}_{*}^{h} \circledast \mathrm{E}_{*}^{p}$ into $\mathbf{C}^{m}$. We have

(1) $m \geqslant h p$.

(2) If $m=h p$, then, up to rigid motions of $\mathbf{C}^{h p}, \tilde{\phi}=\phi \circ \pi$ is given by

$$
\widetilde{\phi}(z, x)=\psi_{z, x}(z, x)=\left(z_{1} x_{1}, \ldots, z_{1} x_{p}, \ldots, z_{h} x_{1}, \ldots, z_{h} x_{p}\right)
$$

Proof: Let $\phi: U \rightarrow \mathbf{C}^{m}$ be a natural $C R$-immersion from an open portion $U$ of $\mathbf{C}_{*}^{h} \circledast \mathbf{E}_{*}^{p}$ into $\mathbf{C}^{m}$. Then Theorem 5.1 implies that, up to rigid motions of $\mathbf{C}^{m}$, the composition $\widetilde{\phi}=\phi \circ \pi$ is given by

$$
\widetilde{\phi}(z, x)=\sum_{j=1}^{h} A^{j}\left(x_{1}, \ldots, x_{p}\right) z_{j},
$$

where $A^{1}\left(x_{1}, \ldots, x_{p}\right), \ldots, A^{h}\left(x_{1}, \ldots, x_{p}\right)$ are mutually orthogonal vector functions of length $|x|$. Moreover, $A^{\mathbf{1}}, \ldots, A^{h}$ satisfy

$$
\begin{aligned}
\left\langle A^{j}, A^{k}\right\rangle & =|x|^{2} \delta_{j k}, \quad\left\langle A^{j}, i A^{k}\right\rangle=0 \\
\left\langle A^{j}, A_{x_{\alpha}}^{k}\right\rangle & =x_{\alpha} \delta_{j k},\left\langle A_{x_{\alpha}}^{j}, A_{x_{\beta}}^{k}\right\rangle=\delta_{j k} \delta_{\alpha \beta}, \\
\left\langle A^{j}, i A_{x_{\alpha}}^{k}\right\rangle & =0, \quad\left\langle A_{x_{\alpha}}^{j}, i A_{x_{\beta}}^{k}\right\rangle=0
\end{aligned}
$$

for $j, k=1, \ldots, h ; \alpha, \beta=1, \ldots, p$.

From (6.36), (6.37) and (6.38) we know that $\left\{A_{x_{\alpha}}^{j}, j=1, \ldots, h ; \alpha=1, \ldots, p\right\}$ is an orthonormal set which spans a totally real $h p$-plane in $\mathbf{C}^{m}$. Therefore, $m \geqslant h p$. This proves statement (1).

If $m=h p$, then (6.37)-(6.38) implies that, for each $j \in\{1, \ldots, h\}, A^{j}$ defines an isometric immersion from an open domain, say $D$, of the Euclidean $p$-space $\mathrm{E}^{p}$ into a totally real $p$-subspace of $\mathrm{C}^{h}$. Moreover, each $A^{j}$ satisfies $\left|A^{j}\right|^{2}=\sum_{\alpha=1}^{p} x_{\alpha}^{2}$. Since, up to rigid motions of $\mathbf{E}^{p}$, the only isometric immersion from the flat $p$-space $D \subset \mathbf{E}^{p}$ into $\mathbf{E}^{p}$ is the inclusion map, each $A^{j}$ must be an inclusion map of $D$ into $\mathbf{E}^{p}$. Furthermore, because $A^{1}, \ldots, A^{h}$ are orthogonal vector functions in $C^{h p}$ which satisfy (6.36)-(6.38), we may choose the complex Euclidean coordinate system $\left\{z_{1}, \ldots, z_{h}\right\}$ on $\mathrm{C}^{h p}$ so that (6.35) takes the following special form of (6.34). This proves statement (2).

REMARK 6.1. Theorem 6.2 can be regarded as the Euclidean version of the natural characterisation of Segre imbeddings obtained in [5].

REMARK 6.2. Further results on convolutions have been obtained in [6]. 


\section{REFERENCES}

[1] A. Bejancu, Geometry of CR-Submanifolds, Mathematics and its Applications 23 (D. Reidel Publishing Co., Dordrecht, 1986).

[2] B.Y. Chen, 'Some $C R$-submanifolds of a Kaehler manifold. I', J. Differential Geom. 16 (1981), 305-322.

[3] B.Y. Chen, 'Some $C R$-submanifolds of a Kaehler manifold. II', J. Differential Geom. 16 (1981), 493-509.

[4] B.Y. Chen, 'Geometry of warped product $C R$-submanifolds in Kaehler manifolds', Monatsh. Math. 133 (2001), 177-195.

[5] B.Y. Chen, 'Geometry of warped products $C R$-submanifolds in Kaehler manifolds. II', Monatsh. Math. 134 (2001), 103-119.

[6] B.Y. Chen, 'More on convolution of Riemannian manifolds', Beiträge Algebra Geom. (to appear).

[7] B. O'Neill, Semi-Riemannian geometry with applications to relativity, Pure and Applied Mathematics 103 (Academic Press, New York, 1983).

Department of Mathematics

Michigan State University

East Lansing, MI 48824-1027

United States of America

e-mail: bychen@math.msu.edu 